\title{
La atención a las necesidades y demandas específicas del alumnado en un mundo globalizado: el caso de un MOOC de español para viajar
}

\section{Attending to the specific needs and demands of students in a globalised world: the case of a 'Spanish for travelling' MOOC}

\author{
Beatriz Sedano Cuevas \\ Universidad Nacional de Educación a Distancia, UNED (España)
}

\section{Resumen}

Los MOOC cuyo contenido está relacionado con la enseñanza de una lengua extranjera (LMOOC), resultan todavía un campo poco explorado, tanto en número de cursos creados como en investigaciones científicas, aunque se encuentra en creciente expansión. Este trabajo presenta la creación y el diseño de un LMOOC dentro del Proyecto Europeo ECO. Está destinado a estudiantes de español que quieren aprender una lengua para viajar, generado a partir de un riguroso análisis de necesidades, según el Enfoque Orientado a la Acción propugnado por el MCERL (Marco Común Europeo de Referencia Para las Lenguas); y siguiendo la tipología propuesta por el proyecto ECO, los SMOOC, cuyo modelo pedagógico está basado en el Conectivismo, e insiste en el componente social, interactivo y ubicuo. El objetivo principal es analizar la atención a las expectativas y necesidades específicas de los participantes que aprenden una lengua con el fin específico de viajar. El estudio sigue una metodología cualitativa a través de cuestionarios realizados al inicio y a la finalización del curso. Los principales resultados indican que un análisis de necesidades previo para el diseño de un LMOOC, con el uso de redes sociales y de REA (Recursos Educativos Abiertos) ayuda en gran medida a cumplir las expectativas de los participantes y a desarrollarse como aprendientes autónomos que podrán llevar a cabo sus acciones de lengua en un contexto globalizado.

Palabras clave: MOOC de lenguas; enseñanza a distancia; aprendizaje social; análisis de necesidades; turismo.

\section{Abstract}

Language MOOCs (LMOOCs), whose content is related to language teaching, is still a field in need of further exploration, with respect to both the number of courses available and scientific research carried out, constituting a developing and expanding field. This paper will show a concrete example of the types of courses developed within the European Project ECO. This course is aimed at Spanish language students who want to learn the language in 
LA ATENCIÓN A LAS NECESIDADES Y DEMANDAS ESPECĆ́FICAS DEL ALUMNADO EN UN MUNDO GLOBALIZADO: EL CASO DE UN MOOC...

order to travel and has been created based on a rigorous needs analysis, according to the approach oriented towards action as advocated by CEFR (Common European Framework of Reference for Languages); and following a new MOOC typology proposed by the ECO project, the sMOOCs, whose pedagogical framework is based on Connectivism, and insists on a social, interactive and ubiquitous component. This study follows a qualitative methodology through initial and final questionnaires. The analysis of data extracted from the first edition of this MOOC will show that a previous needs analysis, with the use of social networks and OER (Open Educational Resources) contribute to fulfil the expectations and development of the participants as autonomous learners who will be able to carry out their actions in the language within a globalised context.

Keywords: Language MOOCs; distance learning; social learning; needs analysis; tourism.

En el contexto actual de un mundo globalizado y tecnológicamente conectado pero todavía lingüísticamente diverso, el conocimiento de una o varias lenguas extranjeras junto a la competencia intercultural son habilidades indispensables para vivir, trabajar o conectarse tecnológicamente y socialmente (Perifanou, 2015; Perifanou y Economides, 2014). A estas competencias hay que sumar las digitales, necesarias también en los contextos de trabajo, vida personal y social tanto a nivel local como regional y global (Pegrum, 2016). Todo esto justifica la necesidad de crear, medir y evaluar iniciativas de enseñanza de segundas lenguas en nuevos formatos como los MOOC (Massive Open Online Courses) y su apoyo en la Web 2.0, ya que presentan un entorno de aprendizaje auténtico, gratuito y colaborativo, abierto a las diferentes situaciones geográficas, temporales o sociales (Sokolik, 2014), que prepara a los estudiantes para ser ciudadanos globales (Dixon y Thomas, 2015). Esto junto a la creciente pero todavía escasa cantidad de trabajos académicos que se ocupan de la enseñanza de segundas lenguas a través de MOOC (Martín Monje y Bárcena, 2014), justifica la relevancia de este artículo, en el que se pretende atender a algunas cuestiones, entre las muchas que existen como objeto de investigación en MOOC (García Aretio, 2015), tales como los métodos pedagógicos y las expectativas de los participantes.

A pesar de que la existencia de los MOOC de lenguas (LMOOC en adelante, siguiendo el término acuñado por Bárcena y Martín-Monje en 2014) todavía se considera "neonatal" (Sokolik, 2014), cada vez son más las plataformas que incluyen MOOC dedicados a la adquisición de una lengua extranjera y que incluyen categorías de "lenguas" en su directorio de búsquedas ("Language" en Edx, "Aprende un idioma" en Coursera, "Language and cultures" en Futurelearn, etc.). Sin embargo, las propuestas en torno a segundas lenguas resultan escasas en comparación con otras disciplinas como la economía, las tecnologías o la ciencia.

Destacan las iniciativas de LMOOC por parte de la UNED desde 2012 a través de su portal UNED Abierta, que incluye no solo MOOC sino cursos OCW (Open Course Ware), y que ha usado diferentes plataformas como OpenMOOC o EdX. En España 
la primera iniciativa que puede considerarse LMOOC fue en 2012 con el MOOC "Inglés profesional/Professional English" (Bárcena, Martín-Monje y Jordano de la Torre, 2016), alojado en dos plataformas: la de UNED, Aprendo/UNED Abierta y la de Telefónica-Universia, Miríada X, con dos ediciones y con casi 50.000 participantes en total, siendo uno de los 3 cursos más populares en la plataforma de la UNED. Asimismo, en la primera fase de la UNED Abierta tuvieron lugar otros LMOOC de inglés, "Empieza con el inglés: aprende las mil palabras más usadas y sus posibilidades comunicativas" y "Starting to write English with no mistakes: level B1“, que ya van por su tercera edición. En 2013, el MOOC de "Alemán para hispanohablantes: nociones fundamentales", con también dos ediciones y más de 40.00o alumnos inscritos en las dos plataformas de UNED-COMA y Miríada X, fue galardonado con el I Premio MECD-Universia/Telefónica al mejor MOOC. Y en la segunda fase de UNED Abierta en 2015 aparecieron un MOOC de español y otro de inglés para el nivel B1, "Español en línea ELE-UNED", y "How to succeed in the English B1 Level Exam", contando este último con dos ediciones y más de 8.000 participantes.

La novedad y potencialidad de los LMOOC demuestra que es necesario establecer el enfoque y la metodología adecuados en consonancia con la enseñanza de segundas lenguas. En el contexto del siglo XXI, el aprendizaje basado en competencias, abierto al mundo exterior y que tiene como base el saber actuar en una lengua de manera social y culturalmente adecuada, parece ser el enfoque más adecuado (Sokolik, 2014). Este enfoque se relaciona con el propuesto por el Marco Común Europeo de Referencia para las Lenguas (MCERL), uno de los puntos de referencia para la globalización de la política educativa de lenguas (Byram y Parmenter, 2012), centrado en la acción y en la competencia comunicativa y funcional, en el que todos los aprendientes de una lengua son "agentes sociales" que tienen que actuar y negociar para conseguir sus objetivos en esa lengua (Consejo de Europa, 2002). Además de los ámbitos profesional y académico, atendidos en las lenguas para fines específicos, los ámbitos personal y público resultan fundamentales en el entorno de un aprendiente de lenguas. En el presente artículo se presenta un LMOOC que atiende en especial a ese ámbito social y al fin específico de aprender una lengua para viajar.

Este LMOOC se inserta dentro del proyecto ECO Learning (The EU-funded project Elearning, Communication and Open-data Massive Mobile, Ubiquitous and Open Learning1), proyecto europeo en el que participan más de 20 instituciones europeas, en el cual se han desarrollado una serie de MOOC (32 hasta la fecha) en seis de los idiomas más demandados de la Unión Europea (inglés, español, alemán, portugués, italiano y francés) con 43.422 participantes hasta la fecha. Este proyecto tiene como objetivo principal extender los MOOC dentro de la Unión Europea y formar a profesores desarrollando sus competencias digitales. Dentro del proyecto, ECO ha tomado una estrategia innovadora ofreciendo a los participantes la creación de su propio MOOC y la distribución del mismo en la plataforma convirtiéndose en e-teachers. En este proyecto solamente dos de los $32 \mathrm{MOOC}$ que se ofertan son 
LA ATENCIÓN A LAS NECESIDADES Y DEMANDAS ESPEĆ́fICAS DEL ALUMNADO EN UN MUNDO GLOBALIZADO: EL CASO DE UN MOOC...

LMOOC, uno de inglés desarrollado por la UNED ("How to succeed in the English B1 Level Exam") y otro de español como lengua extranjera, "Español para viajeros"2, que ocupa la presente investigación, y que se inserta dentro del proyecto de los e-teachers. Este MOOC sigue las tendencias metodológicas del MCERL, con tareas similares a las que tendrán que llevar a cabo en un viaje real a un país hispanohablante.

En el momento del diseño, se ha seguido el modelo pedagógico que propone ECO. Es el denominado sMOOC. La "s" hace referencia por un lado a "social", ya que este modelo se distingue por el componente social de interacción y participación, basado en el Conectivismo, que propugna que el aprendizaje se basa en la construcción de conexiones que se establecen a través de redes en la era digital (Downes, 2007, 2012; Siemens, 2004, 2012); y, por otro lado, a seamless en lengua inglesa, que puede traducirse como un aprendizaje continuo y ubicuo, sin limitaciones, ya que estos cursos deben ser accesibles en diferentes dispositivos, incluidos los móviles e integrarse en la vida real y cotidiana de los participantes atendiendo a las diferentes necesidades especiales de aprendizaje que puedan surgir y a las personas en riesgo de exclusión (Morgado, Teixeira y Jansen, 2015).

En cuanto al análisis de datos, se han examinado los datos de la primera edición con los siguientes objetivos: a) reflexionar sobre la posibilidad de atender en un LMOOC a las demandas específicas de alumnos de diferente procedencia mundial con un propósito específico como es el de aprender una lengua para viajar; b) cómo llevar a cabo el diseño de un curso de estas características con actividades y materiales que satisfagan los diferentes estilos de aprendizaje de los participantes y potencien su desarrollo como aprendientes autónomos. A continuación, se establece el marco teórico que respalda la investigación, se describe la metodología utilizada y se reflexiona sobre los resultados más relevantes obtenidos.

\section{LOS LMOOC EN UN CONTEXTO GLOBALIZADO}

\section{Concepto y desafíos}

Los LMOOC o MOOLC (Perifanou, 2015; Perifanou y Economides, 2014) han sido definidos como cursos de segundas lenguas en línea con acceso no restringido y con una participación potencialmente ilimitada (Martín-Monje y Bárcena, 2014). Frente a los MOOC de otras disciplinas, los LMOOC tienen la dificultad añadida de que el medio de comunicación y el objeto de aprendizaje son el mismo: la lengua extranjera (Martín-Monje, Bárcena y Read, 2014; Ventura, Bárcena y Martín-Monje, 2014). La comunicación lingüística debe ser el eje vertebrador de estos cursos (Sokolik, 2014) y, sin embargo, muchos de los LMOOC existentes han continuado la tradición de los cursos de lenguas en línea que se centraban en la gramática, la redacción escrita o el vocabulario, evitando las complicaciones de la práctica oral en una lengua extranjera y la interacción entre los participantes (Martín-Monje, Bárcena y Read, 2014). 
Autores como Sokolik (2014) proponen un modelo que fomente el intercambio comunicativo y la producción oral, mediante foros que faciliten la subida de audios o vídeos. Es este uno de los mayores desafíos, tanto desde el punto de vista técnico y metodológico, como desde una perspectiva ética y moral (Sokolik, 2014), puesto que el uso de herramientas como Skype, Google Hangout y otras redes sociales como Twitter o Facebook han contribuido a una mayor interacción sincrónica y asincrónica, pero pueden también excluir a potenciales participantes de estos LMOOC por razones geográficas o políticas en lugares donde se restringen esas redes. Además, países en vías de desarrollo donde la alfabetización digital es todavía baja o donde la infraestructura tecnológica es insuficiente también quedan en riesgo de exclusión global (Daniel, Vázquez Cano y Gisbert, 2015). Incluso pueden existir barreras culturales que choquen con la globalización de los modelos pedagógicos de los MOOC, como ocurre en China respecto a la evaluación por pares o los foros de discusión, puesto que el profesor se acepta como única figura educativa (Sharples, et al., 2015).

Una de las mayores controversias y desafíos de los MOOC a nivel global es la cuestión de la evaluación, en especial la evaluación entre pares, la cual suele llevar en muchas ocasiones al abandono del curso debido a la falta de compromiso y de actitud constructiva a la hora de dar feedback a otros participantes, lo que se ha intentado solventar con la utilización de rúbricas o pautas para la evaluación entre pares (Ventura, Bárcena y Martín-Monje, 2014). Este asunto cobra especial relevancia en los LMOOC al existir la problemática del error lingüístico y de la corrección del mismo, además de la disparidad de niveles entre los participantes en el curso (Sokolik, 2014). Es, pues, necesario cambiar la actitud de los participantes en un LMOOC para que adquieran conciencia de la importancia del aprendizaje colaborativo y mostrar los beneficios de este tipo de actividades (Ventura, Bárcena y Martín-Monje, 2014). La solución puede estar en la combinación de diferentes sistemas de evaluación como la comentada evaluación por pares, quizás voluntaria, junto a herramientas más clásicas de evaluación automática tipo test o rúbricas de autoevaluación para llevar a los participantes a reflexionar sobre su propio aprendizaje y progreso (Sokolik, 2014), y nuevas técnicas de evaluación como el uso de portafolios o la evaluación en comunidad, según la influencia que cause la participación de un estudiante en la comunidad (Sánchez-Vera y Prendes-Espinosa, 2015).

\section{Materiales y papel del profesor}

Respecto a los materiales, al igual que todos los MOOC, los LMOOC incluyen principalmente contenido audiovisual, dirigido a la práctica comunicativa en una segunda lengua en un contexto global, con materiales que presenten situaciones comunicativas auténticas, evitando el formato videoconferencia con busto parlante (Sokolik, 2014), con estilo de animación o de duración no superior a 6 minutos (Guo, Kim y Rubin, 2014). 
Asimismo, algunos autores han reflexionado sobre el papel del profesor y su presencia durante el desarrollo de un LMOOC (Castrillo y Martín-Monje, 2015; Castrillo, 2014; Sokolik, 2014). Se señalan la importancia de la presencia activa del profesor en las discusiones para potenciar la comunicación e interacción, o las diferentes funciones que debe cumplir un profesor de un LMOOC en las diferentes fases: diseñador instruccional y creador de contenidos antes del curso, facilitador y evaluador durante el mismo, o investigador al finalizar (Castrillo, 2014). Esta versatilidad exige un alto conocimiento del contexto global en el que está inmersa la lengua que se enseña.

\section{Perfil del estudiante y ventajas de los LMOOC}

El perfil de un participante en un LMOOC no dista del perfil que se ha definido hasta ahora de los MOOC en general: joven, con educación universitaria, estudiante o trabajador activo y que procede normalmente de países desarrollados (Bárcena, Martín-Monje y Read, 2015; Christensen et al., 2013; Martín-Monje y Castrillo, 2016; Rubio, 2016), a pesar de la intención inicial de llegar a aquellos con menos acceso a la educación formal. Por lo tanto, uno de los desafíos en el contexto global es alcanzar de forma real a ese público global, sobre todo a los más vulnerables en riesgo de exclusión social o digital. Por esa razón, algunos autores destacan la importancia de considerar la cuestión de la accesibilidad (Rodríguez-Ascaso, Santos y Boticario, 2015) y las estrategias de inclusión social, y señalan la oportunidad de crecimiento personal que ofrecen los MOOC para personas con problemas educativos, de movilidad geográfica o con restricciones socio-económicas (Rodrigo, 2014).

Dentro del perfil del estudiante de un LMOOC, cabe señalar la cuestión de la motivación. Puesto que, en general, la participación en estos cursos es voluntaria y fruto de un deseo de desarrollo profesional o personal para adaptarse a las necesidades y posibilidades que ofrece un mundo globalizado (Rubio, Fuchs y Dixon, 2016), se puede presuponer que los participantes están en alto grado motivados (Beaven, Codrenau y Creuzé, 2014). Sin embargo, esto no termina de traducirse en tasas de éxito aceptables.

El grado de éxito de un LMOOC es un aspecto que todavía está por determinar y que depende en mayor medida de cada participante puesto que se pueden tener muy diferentes motivaciones y finalidades a la hora de realizarlo, bien sean académicas profesionales o sociales, como pueden ser la de aprender vocabulario y estructuras para manejarse durante un viaje (Sokolik, 2014). Por tanto, una buena definición de los objetivos de estos cursos, así como el unirlo a las necesidades específicas del aprendiz, puede influir positivamente en el aumento del porcentaje de aprobados. No olvidemos que una de las promesas de los MOOC es poner la Educación al alcance de personas en riesgo de exclusión social o profesional, sin duda uno de los retos que plantea la educación en el contexto global del siglo XXI (Martín-Monje y Bárcena, 2014). 
Otros autores (Colpaert 2016; Rubio, Fuchs y Dixon, 2016; Torres y Gago, 2014) señalan que la principal utilidad de los MOOC en el contexto de enseñanza de lenguas es la oportunidad de reunir a estudiantes de diferente procedencia mundial, aparentemente aislados, y crear una auténtica comunidad de aprendizaje en la que los participantes lleven a cabo tareas del mundo reales. Esto se relaciona con otro elemento crucial, que es el de la colaboración y la oportunidad de los MOOC de crear un contexto colaborativo global y una auténtica interacción social. Para llevar a cabo este contexto, hay que considerar el concepto de competencia digital que incluye habilidades como la creatividad, el pensamiento crítico, la resolución de problemas, la colaboración, el trabajo en equipo, la autonomía y la flexibilidad, y las acciones de compartir, reestructurar y mezclar los contenidos procedentes de las redes sociales, desarrollando un papel activo en el panorama global cultural digital (Pegrum, 2016). Estas habilidades junto al enfoque cultural e intercultural, que conducirá a que los participantes se desarrollen como ciudadanos globales con diferentes perspectivas del mundo (Blattner, Dalola y Lomicka, 2015; Heinsch y Rodríguez Pérez, 2015), deben tenerse en cuenta en el diseño de un LMOOC.

\section{Diseño de un LMOOC}

Hasta la fecha parece que no se ha llegado a encontrar un modelo satisfactorio o adecuado para los LMOOC en el contexto globalizado del siglo XXI (Perifanou y Economides, 2014; Perifanou, 2015; Sokolik, 2014), pero sí existen investigaciones y propuestas sobre nuevos modelos (Perifanou, 2015; Teixeira y Mota, 2014), puesto que un diseño adecuado constituye la clave del éxito de un LMOOC (Colpaert, 2014; Perifanou, 2015), el cual debe partir siempre de los objetivos del LMOOC (Sokolik, 2014) y del fin específico del aprendizaje y del contexto local (Colpaert, 2014).

A pesar de las ventajas que parecen presentar los cMOOC o conectivistas para los LMOOC por su filosofía concordante con el enfoque comunicativo para enseñanza de segundas lenguas (Sokolik, 2014), la mayoría de los LMOOC existentes siguen modelos pedagógicos más tradicionales basados en contenidos o en tareas (Heinsch y Rodríguez Pérez, 2015; Rubio, Fuchs y Dixon, 2016). Algunos autores proponen la creación de modelos híbridos con la combinación de lo más adecuado de la metodología de enseñanza de lenguas junto a los dos modelos clásicos: los cMOOC, con sus características sociales implicando a los participantes en la interacción global y el uso real de lengua, y los xMOOC, más similares a los cursos en línea que ofrecen las universidades en su docencia reglada, y que presentan ventajas estructurales a la hora de proveer el fundamento teórico que se necesita en una segunda lengua antes de proceder a la interacción (Bárcena y Read, 2015). A estos puede añadirse la metodología de los MOOC basados en tareas (Lane, 2012), que combinan la pedagogía instructiva y la constructiva con el objetivo principal de realizar tareas similares a las del mundo real (Martín-Monje, Bárcena y Read, 2014), perfecta para 
LMOOC adaptados al contexto global y al enfoque basado en competencias centrado en la acción del MCERL (Consejo de Europa, 2002).

Muchas de las propuestas de diseño para los LMOOC tienden hacia un nuevo modelo de aprendizaje individualizado (Zapata-Ros, 2013), personalizado y adaptativo (Godwin-Jones, 2014; Perifanou, 2015; Teixeira y Mota, 2014), adaptado al perfil y a los intereses individuales de los participantes provocando una mayor implicación en el curso (Torres Mancera y Gago Saldaña, 2014), el cual requiere el desarrollo de herramientas más sofisticadas por parte de educadores, diseñadores y programadores (Daniel, Vázquez Cano y Gisbert, 2015). Este modelo para futuros LMOOC parece el más adecuado a la hora de reunir todas las exigencias de un contexto globalizado y presenta las siguientes características:

- Contextual, cercano a la realidad (Teixeira y Mota, 2014).

- Foco en el alumno a través de un sistema de aprendizaje adaptativo que detecte y se adapte a sus necesidades, progresos y carencias (Perifanou, 2015; Teixeira et al., 2016).

- Aprendizaje autónomo y basado en competencias, con creación de portafolios o evaluación del aprendizaje experiencial (Teixeira y Mota, 2014).

- Con elementos de gamificación. (Teixeira y Mota, 2014).

- Modularidad (Bárcena y Read, 2015; Colpaert, 2014; Godwin-Jones, 2014), escalabilidad (Torres Mancera y Gago Saldaña, 2014) y flexibilidad (Teixeira y Mota, 2014) que permitan a los estudiantes elegir las actividades según sus niveles y necesidades.

- Comunicación auténtica y oral (Rubio, 2016).

- Fuerte componente social, con un aumento progresivo del mismo según va avanzando el dominio del idioma (Godwin-Jones, 2014), con herramientas colaborativas (Perifanou, 2015) y redes sociales externas (Vázquez-Cano y López Meneses, 2015; Read, 2014).

- Ubicuo y adaptado a los dispositivos móviles, de modo que se produzca una auténtica integración del aprendizaje en la vida real y personal (Bárcena y Read, 2015; Godwin-Jones, 2014; Sharples, et al., 2015, Teixeira et al., 2016). 
Figura 1. Características del modelo pedagógico ideal para LMOOC en un contexto global

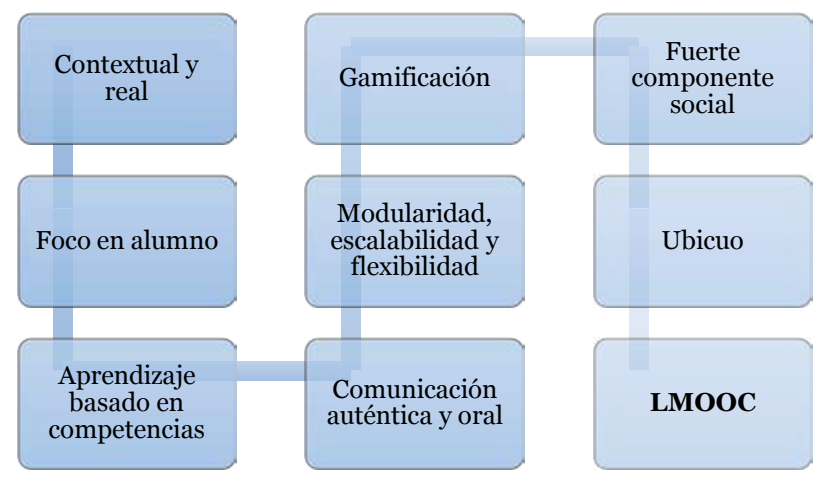

Todos los elementos nombrados anteriormente suponen una propuesta teórica sólida y exhaustiva, pero en la realidad, el profesor que diseña un LMOOC tiene una libertad restringida a la hora de crear las actividades y herramientas de comunicación y evaluación (Read, 2014), ya que no siempre puede elegir la plataforma en la que va a implantar su curso o el tipo de recursos y actividades que dicha plataforma permite implementar, como es el caso del LMOOC en el que se basa esta investigación y que se describe en el siguiente apartado.

\section{METODOLOGÍA}

\section{Análisis de necesidades previo}

El análisis de necesidades se llevó a cabo a través de un estudio cualitativo en el que la recogida de datos se sustentó en cuestionarios a alumnos interesados en este campo específico del español para el turismo $(\mathrm{N}=60)$ y a docentes de español como lengua extranjera (ELE, $\mathrm{N}=100$ ). El primer cuestionario estaba compuesto por una serie de preguntas de escala Likert y abiertas para conocer por parte de estudiantes de español, opiniones, creencias, motivaciones, dificultades, carencias, información sobre estilos de aprendizaje presencial y en línea, en relación con el aprendizaje del español en el contexto de los viajes, y fue completado por estudiantes de hasta 25 nacionalidades distintas. El segundo cuestionario se dirigió a profesionales del español como lengua extranjera de hasta 15 nacionalidades diferentes con la finalidad de analizar la situación actual: grado de necesidad de este tipo de cursos, creencias sobre materiales, situaciones comunicativas más comunes para la situación meta, estilos de aprendizaje, recursos y herramientas o niveles más adecuados para este tipo de cursos. 
La triangulación de datos, fuentes y técnicas se complementó con un análisis pormenorizado de documentos oficiales como el Nivel Umbral, el Marco Común Europeo de Referencia para las lenguas, el Portafolio Europeo de las Lenguas y el Plan Curricular del Instituto Cervantes, además de materiales multimedia y digitales y manuales impresos de ELE y EFE (Español para fines Específicos), con el criterio de definir las características del fin específico del turismo desde el punto de vista del viajero, lo que llevó a identificar los fines y objetivos de este campo, determinar los contenidos que deben ser incluidos en el curso y seleccionar las actividades de aprendizaje, materiales y métodos de evaluación más adecuados.

A continuación, se presenta una tabla que resume las conclusiones de este análisis:

Tabla 1. Análisis de necesidades

\begin{tabular}{|c|c|}
\hline \multicolumn{2}{|c|}{ Análisis de necesidades previo para un curso de español para viajar en línea } \\
\hline Metodología & $\begin{array}{l}\text { - } \quad \text { Cuestionarios (A docentes y estudiantes). } \\
\text { - } \quad \text { Análisis de materiales aprendizaje de documentación: Manuales de ELE y EFE + } \\
\text { Marco Común Europeo de Referencia para las Lenguas + } \\
\text { Plan Curricular del Instituto Cervantes. }\end{array}$ \\
\hline $\begin{array}{l}\text { Cuestionario a docentes } \\
(100)\end{array}$ & $\begin{array}{l}\text { - Creen necesario crear más materiales y cursos específicos } \\
\text { en aprendizaje de ELE para Turismo como ocio. }\end{array}$ \\
\hline $\begin{array}{l}\text { Cuestionario a estudiantes } \\
(61)\end{array}$ & $\begin{array}{l}\text { - Perfil: Procedencia europea sobre todo de Reino Unido, con } \\
\text { idioma materno inglés y mujeres de entre } 20 \text { y } 40 \text { años. } \\
\text { - Creencias y motivaciones: El } 80 \text { \% cree que es } \\
\text { necesario aprender el español cuando viajan a España o } \\
\text { Hispanoamérica y tiene interés por el español para viajar en } \\
\text { modalidad online. } \\
\text { - Carencias: acentos dialectales y vocabulario coloquial. } \\
\text { Estilos de aprendizaje: interacción oral y escrita, vídeos y } \\
\text { películas, actividades interactivas. }\end{array}$ \\
\hline $\begin{array}{l}\text { Situaciones y contextos } \\
\text { más necesarios en un } \\
\text { curso en línea de español } \\
\text { para viajar }\end{array}$ & $\begin{array}{l}\text { - Preguntar y entender sobre direcciones y localizaciones. } \\
\text { Pedir y entender información en un alojamiento, una } \\
\text { tienda, oficina de turismo, en un bar o restaurante. } \\
\text { - Pedir información y comprar billetes de transporte. } \\
\text { - Hablar por teléfono o escribir un correo electrónico para } \\
\text { hacer una reserva en un hotel o restaurante. } \\
\text { - Entender información turística o cultural y las condiciones } \\
\text { de una reserva, viaje, etc. en un catálogo, guía, folleto o } \\
\text { página web. } \\
\text { Entablar una conversación: interactuar con personas } \\
\text { locales. }\end{array}$ \\
\hline
\end{tabular}


LA ATENCIÓN A LAS NECESIDADES Y DEMANDAS ESPECÍFICAS DEL ALUMNADO EN UN MUNDO GLOBALIZADO: EL CASO DE UN MOOC...

\section{Análisis de necesidades previo para un curso de español para viajar en línea}

\begin{tabular}{|c|c|}
\hline $\begin{array}{l}\text { Recursos y herramientas } \\
\text { multimedia más } \\
\text { demandados }\end{array}$ & $\begin{array}{l}\text { - Vídeos sobre cultura hispana y situaciones reales para } \\
\text { viajar. } \\
\text { - Imágenes, infografías y pósteres interactivos. } \\
\text { - Actividades interactivas sobre gramática o vocabulario. } \\
\text { - Textos digitales (periódicos en línea, blogs, etc.). } \\
\text { - Videoconferencias para interacción oral con otros usuarios } \\
\text { de la lengua. }\end{array}$ \\
\hline $\begin{array}{l}\text { Niveles más adecuados } \\
\text { para este tipo de curso }\end{array}$ & $\begin{array}{ll}\text { - } & \mathrm{A} 2 \\
\cdot & \mathrm{B} 1\end{array}$ \\
\hline $\begin{array}{l}\text { Propuesta de } \\
\text { personalización para el } \\
\text { aprendizaje del español } \\
\text { para viajar en línea }\end{array}$ & $\begin{array}{l}\text { Aspectos tecnológicos: } \\
\text { - } \text { Aprendizaje social con Web 2.o (redes sociales, blogs). } \\
\text { - } \text { abiertostos (REA) y MOOC. } \\
\text { - Posibilidad de aprendizaje móvil y ubicuo. } \\
\text { Aspectos de contenido: Centrados en aspectos orales. } \\
\text { Ejemplos: Pedir y entender información en comercios, } \\
\text { tiendas, restaurantes, etc.; Interactuar con la población } \\
\text { local durante el viaje. } \\
\text { Aspectos pedagógicos: Centrados en el uso de herramientas } \\
\text { audiovisuales. }\end{array}$ \\
\hline
\end{tabular}

\section{Descripción del diseño y la metodología del MOOC}

En el curso se plantearon los siguientes objetivos: a) familiarizar a los participantes con las situaciones más comunes que surgen en un viaje e implican el uso de la lengua, proporcionando estructuras y vocabulario útiles, además de cuestiones culturales sobre España para que los alumnos puedan ampliar sus perspectivas y llegar a ser ciudadanos globales (Blattner, Dalola y Lomicka, 2015), usando la lengua y el conocimiento cultural de forma adecuada en sus viajes; b) fomentar el aprendizaje autónomo de la lengua y la cultura española a través del uso de la Web 2.o y las redes sociales; c) practicar de forma colaborativa el español oral y escrito, formando una comunidad global de estudiantes de español que tienen el interés común de viajar a España.

Tal y como se muestra en la tabla 2, el curso se compuso de cuatro módulos para ser realizados en cuatro semanas (uno por semana), pero todos disponibles desde el comienzo del curso con el objetivo de ofrecer una metodología flexible en la que cada estudiante pudiera construir su itinerario de aprendizaje y avanzar a su propio ritmo. 
LA ATENCIÓN A LAS NECESIDADES Y DEMANDAS ESPEĆ́fICAS DEL ALUMNADO EN UN MUNDO GLOBALIZADO: EL CASO DE UN MOOC...

Tabla 2. Estructura del MOOC "Español para viajeros"

\begin{tabular}{|l|l|l|}
\hline Módulo 1 & $\begin{array}{l}\text { Antes del viaje: iHacemos las } \\
\text { maletas! }\end{array}$ & $\begin{array}{l}\text { Planificar un viaje, reservar } \\
\text { alojamiento. }\end{array}$ \\
\hline Módulo 2 & Durante el viaje: Interactuamos & $\begin{array}{l}\text { Situaciones comunicativas para } \\
\text { interactuar en un viaje: direcciones, } \\
\text { restaurante, tiendas, transportes, } \\
\text { gente local, etc. }\end{array}$ \\
\hline Módulo 3 & Personalizamos nuestro viaje & $\begin{array}{l}\text { Tipos de viajes y expresar } \\
\text { preferencias. }\end{array}$ \\
\hline Módulo 4 & Compartimos la experiencia & $\begin{array}{l}\text { Hablar en pasado, hacer } \\
\text { recomendaciones o expresar deseos } \\
\text { futuros respecto a un viaje. }\end{array}$ \\
\hline
\end{tabular}

Los materiales consistieron en una serie de vídeos, grabados por nativos españoles, y material descargable en torno a las situaciones comunicativas que pueden surgir durante un viaje y aspectos culturales del español peninsular. En línea con las directrices del Proyecto ECO, se tuvo en cuenta la accesibilidad, incluyendo subtítulos y transcripciones en los recursos audiovisuales y proporcionando un texto alternativo para las imágenes en los materiales escritos.

Respecto a la evaluación, cada módulo estaba provisto de una actividad de autoevaluación tipo test y una actividad P2P (peer-to-peer), de retroalimentación entre pares, en la que los participantes tuvieron que llevar a cabo una tarea real relacionada con los viajes utilizando materiales auténticos y redes sociales reales. Para la evaluación entre pares, se elaboraron rúbricas con criterios específicos para cada actividad. Cada alumno tenía que evaluar como mínimo a otros dos participantes y a su vez su trabajo era evaluado por dos o más pares, distribución que fue realizada de forma automática por la plataforma.

El componente social y la interacción escrita del curso se consiguieron a través de los foros, uno por cada módulo, y una página de Facebook creada para el curso, en los cuales se plantearon cuestiones de reflexión y de acción comunicativa en torno a los materiales y actividades del curso. Para la interacción oral se propusieron videoconferencias a través de Google Hangout en las que los alumnos pudieron participar voluntariamente. También se realizaron proyectos colaborativos a través de los foros para que los alumnos fueran cocreadores de contenido, como la creación de un glosario final del español para viajeros, el cual fue confeccionado por los participantes del curso a través de un foro en el que debían añadir las palabras y expresiones más útiles que habían aprendido durante el curso. La finalidad de dicha actividad fue la elaboración de un documento único generado a partir de las expresiones propuestas por los participantes, el cual fue puesto a disposición de los alumnos en un repositorio en línea, una vez terminado el curso. Los elementos de gamificación consistieron en la concesión de medallas tras la compleción de módulos 
o actividades p2p y el uso de los votos en los comentarios de los foros a través de la herramienta del "karma".

El aprendizaje autónomo se promovió a través del uso de herramientas existentes en la Web como Pinterest, para que los participantes organizaran los recursos (enlaces, imágenes, vídeos) que se recopilaran durante el curso. En cuanto a la certificación, se ofreció un certificado de participación, así como medallas exportables a Mozilla Open Badges. Estas medallas se traducen en habilidades para definir a los estudiantes como ciudadanos globales e interculturales.

En cuanto a la moderación de las intervenciones de los participantes en la discusión en línea, los profesores creadores del mismo llevaron a cabo la tarea de facilitadores respondiendo a las dudas y animando a los participantes a través de los foros, la herramienta de microblogging y las redes sociales.

\section{Instrumentos y perfil de los participantes}

Para la recogida de datos se siguió una metodología mixta, cuantitativa con los datos proporcionados por la plataforma respecto al progreso y realización de las actividades y módulos, y cualitativa a través de dos cuestionarios, uno inicial y otro final, compuestos por ítems de escala Likert, preguntas cerradas y alguna abierta con el fin de profundizar en las percepciones de los participantes.

El primer cuestionario $(\mathrm{N}=55)$ estaba compuesto por 14 ítems relacionados con el perfil de los participantes, sus estilos de aprendizaje preferidos y dificultades en la lengua, así como sus motivaciones, expectativas e intenciones respecto al curso. El segundo cuestionario $(\mathrm{N}=41)$ lo formaban 37 ítems con cuestiones relacionadas con la percepción de los participantes sobre expectativas cumplidas, adecuación de los contenidos, actividades y metodología del curso.

Según los datos extraídos de la plataforma, en la primera edición de este curso se registraron 209 participantes, de los cuales 147 empezaron el curso (el 17\% aprobaron el curso). Respecto al perfil, la mayor parte de los participantes eran adultos jóvenes, (29,6\% entre 30 y 40 años y $25,9 \%$ entre 20 y 30 años), mujeres $(77,8 \%)$, con educación universitaria o de posgrado (73\%) y la mayoría activos laboralmente (75,6\%) o estudiantes (14,6\%), lo que coincide con el perfil del participante MOOC ya establecido (Martín-Monje y Castrillo, 2016). En cuanto a la procedencia de los participantes fue muy variada, puesto que en los dos cuestionarios se registraron hasta 22 nacionalidades distintas de procedencias muy diversas del globo, siendo la mayoría británica, seguida de estadounidense y otras nacionalidades europeas.

\section{Preguntas de investigación}

El principal objetivo de este estudio es analizar el grado de cumplimiento de las expectativas, necesidades y demandas de los participantes de procedencia global 
LA ATENCIÓN A LAS NECESIDADES Y DEMANDAS ESPEĆ́fICAS DEL ALUMNADO EN UN MUNDO GLOBALIZADO: EL CASO DE UN MOOC...

diversa en un LMOOC centrado en un fin específico, como es el de aprender una lengua para viajar, y en qué medida el diseño y la metodología del curso responden a ese propósito. Las preguntas de investigación son las siguientes:

1. ¿Es posible atender a las expectativas y necesidades específicas de los participantes en un LMOOC en un contexto globalizado?

2. ¿Son adecuadas la metodología y actividades propuestas en este LMOOC para un curso de español para viajar?

\section{RESULTADOS Y DISCUSIÓN}

En esta sección se analizan los resultados relacionados con dos temas fundamentales: a) las expectativas y el cumplimiento de las mismas por parte de los participantes en un MOOC de lenguas con un fin específico; b) la adecuación de los materiales y la metodología para un curso masivo y en línea con el fin específico de aprender una lengua para viajar.

\section{Respuesta a la pregunta 1}

¿Es posible atender a las expectativas y necesidades específicas de los participantes en un LMOOC en un contexto globalizado?

Del cuestionario inicial se consideraron losítems relacionados con las expectativas de aprendizaje, los deseos, las carencias y las motivaciones. Las expectativas de los participantes antes de iniciar el curso eran bastantes realistas en cuanto a que el nivel con que se describía la mayoría coincidía con el nivel para el cual estaba diseñado el curso, A2-B1 (55,6\%), mientras que un $25,5 \%$ correspondía a B2 y solo un $7,4 \%$ al nivel A1.

Respecto a los deseos y carencias, los participantes deseaban mejorar su vocabulario (65,5\%), la expresión e interacción orales $(61,8 \%)$, la expresión e interacción escritas $(49,1 \%)$ y la comprensión auditiva (47,3\%). Sin embargo, resultó llamativo el poco interés por parte de los estudiantes en participar en videoconferencias sincrónicas con herramientas externas (Google Hangout) para practicar las destrezas orales. En cuanto a los contenidos que los participantes consideraban fundamentales para un curso de español para viajar, coincidieron en muy alto grado con los contenidos del curso: vocabulario, información cultural sobre España, situaciones comunicativas como reservar, comprar, alquilar un coche, entender direcciones, interactuar con la gente local, etc.

En cuanto a la motivación, las razones por las que se inscribieron en el curso eran variadas: el 41,8\% quería mejorar su nivel general, seguido del 20,4\% que deseaba practicar de forma diferente a través de la modalidad en línea, mientras que un $26 \%$ lo hizo porque le interesaba viajar en español o tiene la finalidad de viajar a España 
en el futuro. Las intenciones de los participantes antes de empezar el curso también fueron diversas: un 32,7\% solamente quería comprobar su nivel de español y ver qué entendía, mientras que un 23,6\% tenía la intención de completar el curso. Este dato es importante, puesto que el porcentaje de finalización del curso fue el $17 \%$, por lo que la gran mayoría de los que se habían propuesto finalizar el curso lo consiguieron.

En el segundo cuestionario se analizaron los ítems que hacen referencia al cumplimiento de expectativas. Al finalizar el curso, la percepción sobre el aprendizaje del MOOC por parte de los participantes fue muy positiva. De este modo, el 87,8\% consideró que había aprendido mucho o bastante, el 95,1\% vio cumplidas sus expectativas y el 97,6\% pensaba que el curso cumplía con los objetivos (véase figura 2). Además, el $97,5 \%$ consideró que el curso cubría los contenidos necesarios para viajar y el 92,7\% tuvo la convicción de que podrá aplicar lo aprendido en el curso en su vida personal y ser un viajero independiente (véase figura 3 ).

Figura 2. Expectativas cumplidas de los participantes
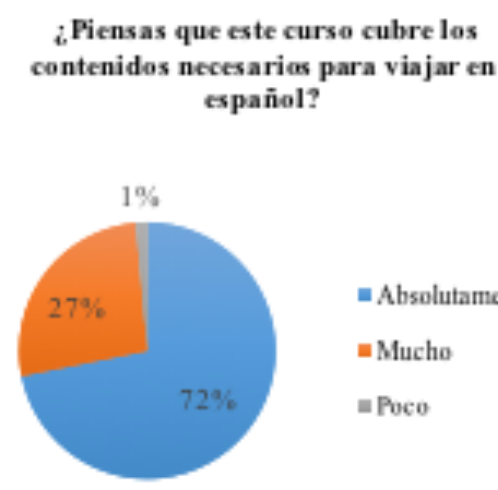
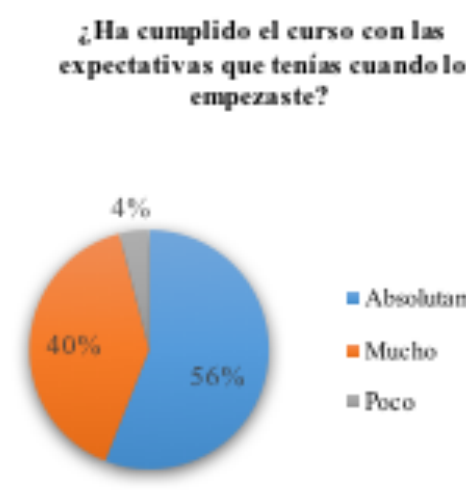

- Abevlutamento

- Mucho

ace 
LA ATENCIÓN A LAS NECESIDADES Y DEMANDAS ESPEĆ́FICAS DEL ALUMNADO EN UN MUNDO GLOBALIZADO: EL CASO DE UN MOOC...

Figura 3. Expectativas cumplidas de los participantes

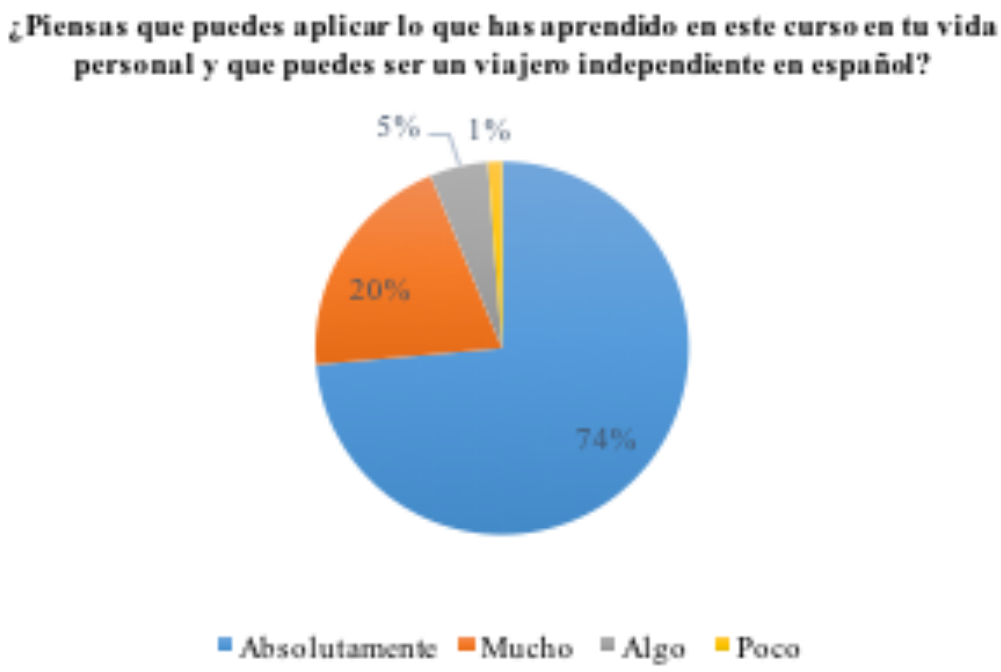

\section{Respuesta a la pregunta 2}

¿Son adecuadas la metodología y actividades propuestas en este LMOOC para un curso de español para viajar?

En esta pregunta se consideraron los ítems de los dos cuestionarios relacionados con los materiales, las actividades y la evaluación.

En el cuestionario inicial, dos de los 14 ítems respondían a estas cuestiones para conocer los estilos de aprendizaje preferidos por los participantes antes del curso. Uno de ellos se refería a los estilos de aprendizaje y herramientas preferidas, que son la conversación e interacción oral (61,8\%), escuchar audios (60\%), ver vídeos $(58,2 \%)$ o leer sobre temas de interés $(52,7 \%)$. Esto coincidía con la metodología del curso basada en el material audiovisual sobre situaciones comunicativas de interacción en un viaje.

En el cuestionario final se recogieron las percepciones de los participantes sobre estas cuestiones. En cuanto a los materiales, el 97,5\% consideró que los materiales y actividades eran interesantes y adecuados para viajar (véase figura 4). Sobre las diferentes herramientas (vídeos, subtítulos y transcripciones, material descargable, material complementario como enlaces o contenido de la página de Facebook), todas las respuestas tuvieron una percepción destacablemente positiva. Al ser preguntados por posibles mejoras en el curso, los alumnos sugirieron la utilización de una 
herramienta síncrona de chat escrito y oral -aunque tal y como se ha indicado, cuando se les dio la posibilidad con Google Hangout no la aprovecharon.

Figura 4. Percepción sobre los materiales y contenidos del curso

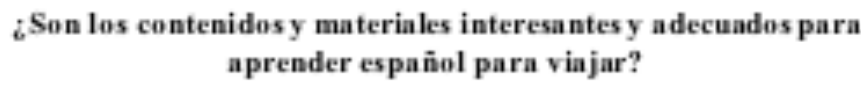

aprender español para viajar?

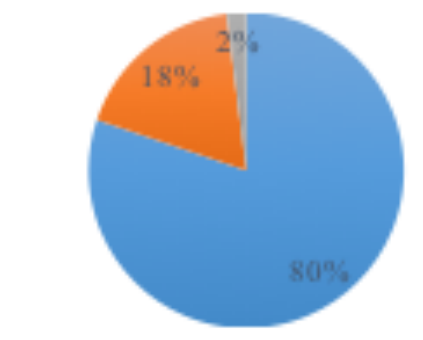

- Absoluntamente $\mathbf{m u c h o}_{\text {Maco }}$

Respecto a las actividades y metodología de evaluación, la evaluación de test con corrección automática recibió mejor valoración (96\%, "muy buena" o "buena) que la evaluación por pares, pero esta última todavía se mantuvo en un grado bastante positivo (78\%, "muy buena" o "buena"). Aun así, todavía los alumnos no se sintieron plenamente cómodos valorando el trabajo de sus compañeros en las actividades P2P. Algunos testimonios lo corroboran: "Pienso que la idea [...] es buena, pero en realidad es muy difícil de hacer. No me sentía cómodo valorando los esfuerzos de otros", o "No me gusta evaluar a otros estudiantes".

\section{CONCLUSIÓN}

Este artículo ha querido presentar algunas cuestiones relevantes con los MOOC de lenguas o LMOOC y su relación con un mundo globalizado: características y elementos, dificultades y desafíos, así como metodologías y modelos pedagógicos más adecuados. Se ha presentado el diseño y el desarrollo de un LMOOC concreto desarrollado dentro del Proyecto Europeo ECO, siguiendo la filosofía y el modelo pedagógico específico propuesto, sMOOC, con el foco en el componente social y teniendo en cuenta cuestiones de inclusión y accesibilidad. El objetivo del estudio ha sido analizar la posibilidad de atender a las demandas específicas y cumplir las expectativas de los participantes en un LMOOC orientado a un fin específico, como es en este caso, el del español con el fin de viajar. 
LA ATENCIÓN A LAS NECESIDADES Y DEMANDAS ESPEĆ́fICAS DEL ALUMNADO EN UN MUNDO GLOBALIZADO: EL CASO DE UN MOOC...

Los resultados muestran que, en general, las expectativas de los participantes se cumplen en cuanto a sus objetivos, los objetivos del curso y sus deseos o creencias de lo esperado en un curso de estas características. Además, las actividades, materiales y metodología parecen satisfacer en alto grado a los participantes. Sin embargo, se ha visto que hay algunos elementos que deberían ser mejorados en futuras ediciones, como los elementos de interacción oral y las herramientas adaptativas o personalizadas para que el aprendizaje de la lengua sea adecuado al contexto de cada participante (Godwin-Jones, 2014; Perifanou, 2015; Teixeira y Mota, 2014). En futuras investigaciones se pretende analizar el grado de participación e interacción social en LMOOC como uno de los objetivos principales de este tipo de cursos (Perifanou, 2015) teniendo en cuenta las ventajas y limitaciones de que estas se realicen en una segunda lengua.

Como conclusión general, puede afirmarse que la creación de un curso masivo de lenguas a partir de un análisis de necesidades previo, junto con la elección de esta tipología de sMOOC (social e ubicuo), con el uso de redes sociales, REA (Recursos Educativos Abiertos) y actividades de revisión por pares (P2P), ayuda a cumplir las expectativas de los participantes que poseen una finalidad específica de aprendizaje y favorece en gran medida a que los participantes se desarrollen como aprendientes autónomos capaces de actuar como agentes sociales (Consejo de Europa, 2002) y mejoren su competencia comunicativa en el campo específico del turismo como viajeros.

\section{NOTAS}

1. http://project.ecolearning.eu/es/

2. https://hub11.ecolearning.eu/course/espanol-para-viajeros/

\section{REFERENCIAS BIBLIOGRÁFICAS}

Bárcena, E., y Martín-Monje, E. (2014). Introduction. Language MOOCs: An emerging field. En E. Martín-Monje y E. Bárcena, (Eds.), Language MOOCs: Providing learning, transcending boundaries. (1-15). Berlin: De Gruvter Open. Recuperado de http:// www.degruvter.com/viewbooktoc/ product/455678

Bárcena, E., Martín-Monje, E., y Jordano de la Torre, M. (2016). Innovación metodológica y tecnológica en la enseñanza del inglés para turismo a distancia. Ibérica: Revista de la
Asociación Europea de Lenguas para Fines Específicos (AELFE), 31, 39-62. Recuperado de http://www.aelfe.org documents/31 02 IBERICA.pdf

Bárcena, E., Martín-Monje, E., y Read, T. (2015). Potentiating the human dimension in Language MOOCs. Proceedings of the European Stakeholder Summit on experiences and best practices in and around MOOCs, EMOOCs 2015. (46-54). Mons, Belgium: Université Catholique de Louvain. Recuperado de http://www. emoocs2015.eu/sites/default/files Papers.pdf 
Bárcena, E., y Read, T. (2015). The Role of Modularity and Mobility in Language MOOCS. Verbeia, Revista de estudios filológicos, $o$, 28-35. Madrid: Universidad Camilo José Cela. Recuperado de http:// www.ucic.edu/book/verbeia-revista-deestudios-filologicos-no-o/

Beaven, T., Codreanu, T., y Creuzé, A. (2014). Motivation in a Language MOOC: Issues for Course Designers. En E. Martín-Monje y E. Bárcena, (Eds.), Language MOOCs: Providing learning, transcending boundaries (48-66). Berlin: De Gruyter Open. Recuperado de http:// www.degruyter.com/viewbooktoc/ product $/ 455678$

Blattner, G., Dalola, A., y Lomicka, L. (2015). Tweetsmarts: A Pragmatic Analysis of Well Known Native French Speaker Tweeters. En E. Dixon y M. Thomas (Eds.), Researching Language Learner Interaction Online: From Social Media to MOOCs. CALICO Monograph Series, 13, (213-236). Recuperado de http://calico. org/LearnerInteractionsOnline.pdt

Byram, M. S., y Parmenter, L. (2012). The Common European Framework of Reference: The Globalisation of Language Education Policy. Bristol: Multilingual Matters.

Castrillo de Larreta-Azelain, M. D. (2014). Language Teaching in MOOCs: The Integral Role of the Instructor. En E. Martín-Monje y E. Bárcena, (Eds.), Language MOOCs. Providing Learning, Transcending Boundaries. (67-90). Berlin: De Gruvter Open. Recuperado de http://www.degruyter.com/ viewbooktoc/product/455678

Castrillo, M. D., y Martín-Monje, E. (2015). Improving quality of teaching in MOOCs: A practical analysis of the new instructor role in the sMOOC model proposed by the European ECO Project (eLearning, Communication and open data: Massive, mobile, ubiquitous and open Learning).
Proceedings of EDULEARN 2015 (133141). Barcelona, Spain: IATED Academy.

Colpaert, J. (2014). Conclusion. Reflections on present and future: Towards an ontological approach to LMOOCs. En E. Martín-Monje y E. Bárcena, (Eds.), Language MOOCs: Providing learning, transcending boundaries (161-172). Berlin: De Gruyter Open. Recuperado de http://www.degruyter.com/ viewbooktoc/product/455678

Colpaert, J. (2016). Foreword. En E. MartínMonje, I. Elorza y B. García Riaza. (Eds.), Technology-Enhanced Language Learning for Specialized Domains (9-22). London, United Kingdom: Routledge

Christensen G., Steinmetz, A., Alcorn, B., Bennett, A., Woods, D., y Emanuel, E. J. (2013). The MOOC Phenomenon: Who takes Massive Open Online Courses and Why. Social Science Electronic Publishing. doi: http://dx.doi. org/10.2139/ssrn.2350964

Consejo de Europa (2002). Marco común europeo de referencia para las lenguas: aprendizaje, enseñanza, evaluación. Madrid: Instituto Cervantes, Ministerio de Educación, Cultura y Deporte, Anaya.

Daniel, J., Vázquez Cano, E., y Gisbert, M. (2015). El futuro de los MOOC: ¿aprendizaje adaptativo o modelo de negocio? RUSC. Universities and Knowledge Society Journal, 12(1), 6474. doi: http://dx.doi.org/10.7238/rusc. v12i1.2475

Dixon, E., y Thomas, M. (2015). Introduction. En E. Dixon y M. Thomas (Eds.), Researching Language Learner Interaction Online: From Social Media to MOOCs, CALICO Monograph Series, 13, (1-8). Recuperado de http://calico.org/ LearnerInteractionsOnline.pdf

Downes, S. (2007, 3 de febrero). What Connectivism is [Blog]. Recuperado de http://halfanhour.blogspot.com. es/2007/02/what-connectivism-is.htm 
LA ATENCIÓN A LAS NECESIDADES Y DEMANDAS ESPEĆ́fICAS DEL ALUMNADO EN UN MUNDO GLOBALLZADO: EL CASO DE UN MOOC...

Downes, S. (2012, 6 de enero). Creating the Connectivist Course. [Blog]. Recuperado de http://halfanhour.blogspot.com. es/2012/01/creating-connectivist-course. html

García Aretio, L. (2014). MOOC: ¿tsunami, revolución o moda pasajera? RIED. Revista Iberoamericana de Educación A Distancia, 18(1), 9-21. doi: http://dx.doi. org/10.5944/ried.18.1.13812

Godwin-Jones, R. (2014). Global reach and local practice: The promise of MOOCS. Language Learning \& Technologu, 18(3), 5-15. Recuperado de http://llt.msu.edu/ issues/october2014/emerging.pdf

Guo, P., Kim J., y R. Rubin (2014). How Video Production Affects Student Engagement: An Empirical Study of MOOC Videos. Proceedings of ACM Conference on Learning at Scale, (41-70) doi: http:/ dx.doi.org/10.1145/2556325.2566239

Heinsch, B., y Rodríguez Pérez, M. N. (2015). MOOC: un nuevo escenario de enseñanzaaprendizaje de lenguas extranjeras. @ tic. Revista d'innovació educativa, 14(1), 1-12. Recuperado de https://ojs.uv.es/ index.php/attic/article/view/4315

Lane, L. M. (2012, August 15). Three kinds of MOOCs. [Blog]. Recuperado de http://www.lisahistory.net wordpress/2012/08/three-kinds-ofmoocs

Martín-Monje, E., Bárcena, E. y Read, T. (2014). Peer-to-peer interaction and linguistic feedback in foreign language MOOCS. Revista de currículum y formación del profesorado, 18(1), 167183. Recuperado de http://recvt.fecvt. es/index.php/profesorado/article/ view/42906

Martín-Monje, E., y Castrillo, M. D. (2016). A redefinition of the teacher and student roles in Language MOOCs: The example of "How to succeed in the English-B1 Level Exam". Paper presented at the LLAS 11th annual e-learning Symposium 2016. Southampton, United Kingdom
Martín-Monje, E., y Bárcena, E. (Eds.). (2014) Language MOOCs: Providing learning, transcending boundaries. Berlin: De Gruyter Open. Recuperado de http:/ www.degruyter.com/viewbooktoc/ product/455678

Morgado, L., Teixeira, A., y Jansen, D. (2015). D2.3 Instructional design and scenarios for MOOCs -version 2. ECO Project Deliverable. Recuperado de http://project.ecolearning.eu/wpcontent/uploads/2016/03/ECO D2.3 Instructional design and scenarios for MOOCs v1.0.pdf

Pegrum, M. (2016). Languages and literacies for digital lives. En E. Martín-Monje, I. Elorza y B. García Riaza. (Eds.), Technology-Enhanced Language Learning for Specialized Domains (9-22). London, United Kingdom: Routledge

Perifanou, M. (2015). Personalized MOOCs for Language Learning: A challenging proposal. ELearning Papers, 45. Recuperado de http:/ openeducationeuropa.eu/sv/ node/174128

Perifanou M., y Economides, A. (2014) MOOCs for foreign language learning: an effort to explore and evaluate the first practices. In Proceedings of the INTED2014. Conference held in Valencia Spain (8-12 March 2014). Recuperado de http://library.iated.org/view/ PERIFANOU2014MOO

Read, T. (2014). The architectonics of MOOCs. En E. Martín-Monje y E. Bárcena, (Eds.), Language MOOCs: Providing Learning, Transcending Boundaries. (91-105). Berlin: De Gruyter Open. Recuperado de http://www.degruyter. com/viewbooktoc/product/455678

Rodríguez-Ascaso, A., y González Boticario J. (2015). Accesibilidad y MOOC: Hacia una perspectiva integral. RIED. Revista Iberoamericana de Educación A Distancia, 18(2), 61-85. doi: http:/D dx.doi.org/10.5944/ried.18.2.13670 
Rodrigo, C. (2014). Accessibility in Language MOOCs. En E. Martín-Monje y E. Bárcena, (Eds.), Language MOOCs: Providing Learning, Transcending Boundaries. (106-126). Berlin: De Gruyter Open. Recuperado de http://www.degruyter. com/viewbooktoc/product/455678

Rubio, F., Fuchs, C., y Dixon, E. (2016). MOOCs better by design. En E. MartínMonje, I. Elorza, y B. García Riaza. (Eds.), Technology-Enhanced Language Learning for Specialized Domains. (177-188). London, United Kingdom: Routledge.

Sánchez-Vera, M. M., y Prendes-Espinosa, M. P. (2015). Más allá de las pruebas objetivas y la evaluación por pares: alternativas de evaluación en los MOOC. RUSC. Universities and Knowledge Society Journal, 12(1), 119-131. doi hittp:// dx.doi.org/10.7238/rusc.v12i1.2262

Sharples, M. et al., (2015). Mobile and Accessible Learning for MOOCs. Journal of Interactive Media in Education. 2015(1), 4. doi: http://doi.org/10.5334/ ime.ai

Siemens, G. (2004). Conectivismo: Una teoría de aprendizaje para la era digital. Recuperado de https://es.scribd.com/ doc/201419/Conectivismo-una-teoriadel-aprendizaje-para-la-era-digital

Siemens, G. (3 de junio de 2012). What is the theory that underpins our MOOCs? [Blog]. Recuperado de http://www. elearnspace.org/blog/2012/06/03/whatis-the-theory-that-underpins-our-moocs

Sokolik, M. (2014). What constitutes an effective Language MOOC. En E. Martín-Monje y E. Bárcena, (Eds.), Language MOOCs: Providing Learning, Transcending Boundaries. (16-32). Berlin: De Gruyter Open. Recuperado de http://www.degruyter.com/ viewbooktoc/product/455678

Teixeira, A., y Mota, J. (2014). A proposal for the methodological design of collaborative language MOOCs. En E. Martín-Monje y E. Bárcena, (Eds.), Language MOOCs: Providing Learning, Transcending Boundaries. (33-47). Berlin: De Gruvter Open. Recuperado de http:/ www.degruyter.com/viewbooktoc/ product/455678

Teixeira, A., García-Cabot, A., García-López, E., Mota, J. y de-Marcos, L. (2016). A new competence-based approach for personalizing MOOCs in a mobile collaborative and networked environment. RIED. Revista Iberoamericana de Educación a Distancia, 19(1), 143160. doi: http://dx.doi.org/10.5944/ ried.19.1.14578

Torres, D., y Gago, D. (2014). Los MOOC y su papel en la creación de comunidades de aprendizaje y participación. RIED. Revista Iberoamericana de Educación a Distancia, 17 (1), 13-34. doi: http:/ revistas.uned.es/index.php/ried/article/ view/11570

Vázquez-Cano, E., y López Meneses, E. (2015). La filosofía educativa de los MOOC y la educación universitaria. RIED. Revista Iberoamericana de Educación a Distancia, 18(2), 25-37. doi: http:// dx.doi.org/10.5944/ried.18.2.14261

Ventura, P., Bárcena, E., y Martín-Monje, E. (2014). Analysis of the impact of social feedback on written production and student engagement in Language MOOCs. Procedia- Social and Behavioural Sciences, 141, 512-517. Recuperado de http://www.sciencedirect.com/science/ article/pii/S1877042814035137

Zapata-Ros, M. (2013). MOOCs, una visión crítica y una alternativa complementaria: La individualización del aprendizaje y de la ayuda pedagógica. E-LIS, E-prints in Library and Information Science. Recuperado de http://eprints.rclis. org/18658 


\section{PERFIL ACADÉMICO Y PROFESIONAL DE LA AUTORA}

Beatriz Sedano Cuevas. Licenciada en Filología Hispánica con un Máster en Lingüística Aplicada a la Enseñanza del Español para extranjeros. Docente de ELE desde hace 10 años, está actualmente realizando su tesis doctoral dentro del Programa de Doctorado de TIC en la Enseñanza y Tratamiento de Lenguas de la UNED. Sus líneas de investigación principales son el diseño curricular de cursos de español para fines específicos, las TIC en enseñanza de lenguas y los MOOC. E-mail: beatrizsedanocuevas@gmail.com

\section{DIRECCIÓN DE LA AUTORA}

Calle Salitre $154^{\circ} \mathrm{D}$

28012 Madrid, España

Fecha de recepción del artículo: 01/06/2016

Fecha de aceptación del artículo: 04/08 /2016

\section{Como citar este artículo:}

Sedano Cuevas, B. (2017). La atención a las necesidades y demandas específicas del alumnado en un mundo globalizado: el caso de un MOOC de español para viajar. RIED. Revista Iberoamericana de Educación a Distancia, 2O(1), pp. 161-182. doi: http://dx.doi.org/10.5944/ried.20.1.16692 